\title{
Neuroleptic Malignant Syndrome Induced by Olanzapine in a Patient with Huntington's Disease
}

\author{
Jose Luis López-Sendón Moreno ${ }^{\mathrm{a}, *}$, Jose María Palau Fayos ${ }^{\mathrm{b}}$, Alberto Díaz de Santiago ${ }^{\mathrm{b}}$ \\ and Justo García de Yébenes ${ }^{\mathrm{a}}$ \\ ${ }^{a}$ Servicio de Neurología, Hospital Ramón y Cajal, Madrid, Spain \\ ${ }^{\mathrm{b}}$ Servicio de Medicina Interna, Hospital Puerta de Hierro, Madrid, Spain
}

Olanzapine is an atypical antipsychotic that binds to a large number of neurotransmitter receptors, including the dopamine $\mathrm{D}_{1}, \mathrm{D}_{2}$, and $\mathrm{D}_{4}$, serotonin and histamine receptors. Its antipsychotic effects are likely the result of potent antagonism at dopamine and serotonin receptors [1]. It is frequently used in Huntington's disease (HD) since it reduces chorea, is a mood stabilizer, augments antidepressants and also encourages weight gain [2]. Olanzapine has probably a better evidence base than the other atypical antipsychotic drugs [3] (with the exception of clozapine) including beneficial effects on the gait disorder and the psychiatric disturbance of HD. Its side effects should always be balanced before starting treatment and include excessive sedation, parkinsonism, tardive dyskinesia and negative metabolic side effects.

Neuroleptic malignant syndrome (NMS) has been reported with the use of dopamine depleting agents and other dopamine blockers in HD [4] but to our knowledge, it has never been reported with olanzapine use in patients with HD.

*Correspondence to: Jose Luis López-Sendón Moreno, Servicio de Neurología, Hospital Ramón y Cajal, Carretera de Colmenar Km9,100, Postal Code: 28034, Madrid, Spain. Tel.: +34 913368821; E-mail: joselopezsendon@ hotmail.com.
We present the case of a NMS developing in a HD patient treated with olanzapine. The patient is a 43 year old male who developed mild chorea, bradikinesia and rigidity with progressive psychiatric abnormalities around age 30 . His father died of HD. The clinical diagnosis was confirmed with molecular testing (49 CAG triplets). Several months ago the patient developed aggressiveness against his relatives and caretakers and became rigidly perseverative with occasional psychotic delusions and olanzapine, $10 \mathrm{mg} / \mathrm{d}$, was prescribed. The patient developed severe parkinsonism and dysphagia and the dose of olanzapine was reduced to $5 \mathrm{mg} / \mathrm{d}$. In addition, diazepam, $5 \mathrm{mg} / \mathrm{tid}$, and escitalopram, $10 \mathrm{mg} / \mathrm{d}$, were prescribed for anxiety and depression respectively.

Three months after the last change of medication the patient presented with hypertermia, $39.5^{\circ} \mathrm{C}$, tachycardia, excessive sweating, severe rigidity and parkinsonian signs. Chest radiographs, urine sediment and blood cultures were normal. A lumbar puncture revealed no abnormalities. Laboratory chemistries showed increased creatine kinase (CK) levels (2742 IU/L) and hypernatremia (Na $155 \mathrm{mEq} / \mathrm{L})$ while the haematological values were normal. A transthoracic echocardiography revealed no abnormalities. No source of infection was found. Two doses of wide 
spectrum antibiotics and antipyretics (paracetamol) had been administered empirically without improvement. Antibiotics and olanzapine were discontinued and intravenous hydration started while no additional medication was administered. This resulted in a gradual improvement and three days later his fever had subsided while the levels of $\mathrm{CK}$ and sodium had normalized. The patient was discharged and two weeks later remained in his basal clinical situation.

NMS is a potentially fatal drug reaction that has been associated with the use of antypsychotics. Several diagnostic criteria for NMS are in use. Whilst there is agreement regarding the general symptoms and signs that may occur as part of NMS, there is disagreement over which of these should be considered primary features of the disorder. Classical NMS is characterized by two symptoms: rigidity and hyperthermia as well as laboratory finding of an elevated CK. Additional symptoms may include abnormal mental status, akinesia, autonomic instability, myoclonus, chorea or generalized tonic-clonicseizures [5, 6]. Incidence estimates for NMS vary widely, and it remains uncertain whether atypical drugs are less likely to cause NMS than the typical antypsichotics. Historically, the prevalence of NMS has been estimated to be between $0.5 \%$ and $3.2 \%$. These data was obtained only with high potency antipsychotics (i.e., haloperidol and fluphenazine). However, recent prospective trials estimate the prevalence with newer low-potency agents to be between 0.02 " and $1.4 \%$ [7].

It has been described in patients with Parkinson's disease [8], especially after sudden suppression of the dopaminergic treatment, including the suppression of the DOPA-rich VitiaFaba beans [9]. All this data suggests that this syndrome is not only related to pharmacological blockade of dopamine receptors but to other mechanisms of acute de-compensation of dopamine neurotransmission.

Most patients with HD have abnormal dopamine neurotransmission which is present from the initial stages of the disease and is more severe in patients with the juvenile form [10]. Therefore these patients are at special risk of NMS when treated with antipsychotics, although the occurrence of this complication with atypical anti-psychotics is rarely reported [11]. Olanzapine been reported to produce NMS in schizophrenic patients [12] but, to our knowledge, this is the first description of such complication in patients with HD.

Behavioral interventions and potentially safer treatments should be considered as a first line treatment for behavioural problems in HD patients. However, when atypical antypsichotics are used, it is important to consider such diagnosis when they develop clinical symptoms compatible with NMS.

\section{POTENTIAL CONFLICT OF INTEREST}

There is no potential conflict of interest from each author to the research covered in the article submitted.

\section{FINANCIAL DISCLOSURES}

The authors have no financial disclosures or nothing to disclose.

\section{REFERENCES}

[1] Maloney AE, Sikich L. Olanzapine approved for the acute treatment of schizophrenia or manic/mixed episodes associated with bipolar I disorder in adolescent patients. Neuropsychiatric Disease and Treatment. 2010;6:749-66.

[2] Grove VE Jr, Quintanilla J, DeVaney GT. Improvement of Huntington's disease with olanzapine and valproate. N Engl J Med. 2000;343:973-4

[3] Phillips W, Shannon KM, Barker RA. The Current Clinical Management of Huntington's Disease. Mov Disord. 2008;23(11):1491-504.

[4] Burke RE, FahnS S, Mayeux R, Weinberg H, Louis K, Willner JH. Neuroleptic malignant syndrome caused by dopamine-depleting drugs in a patient with Huntington's disease. Neurology. 1981;31(8):1022-25.

[5] Trollor JN, Chen X, Sachdev PS. Neuroleptic Malignant Syndrome associated with atypical antipsychotic drugs. CNS Drugs. 2009;23(6):477-92.

[6] Medication-induced movement disorders: Neuroleptic malignant syndrome. Diagnostic and Statistical Manual of Mental Disorders: DSMN-IV-TR. Washington D.C.: American Psychiatric Association; 2000;795-98.

[7] Hall R, Hall R, Chapman M. Neuroleptic malignant syndrome in the elderly: diagnostic criteria, incidence, risk factors, pathophysiology and treatment. Clinical Geriatrics. 2006;14(5):39-46.

[8] Mizuno Y, Takubo H, Mizuta E, Kuno S. Malignant syndrome in Parkinson's disease: concept and review of the literature. Parkinsonism Relat Disord. 2003;9Suppl 1:S3-9.

[9] Ladha SS, Walker R, Shill HA. Case of neuroleptic malignant-like syndrome precipitated by abrupt fava bean discontinuance. Mov Disord. 2005;20:630-1.

[10] Sánchez-Pernaute R, Künig G, del Barrio Alba A, de Yébenes JG, Vontobel P, Leenders KL. Bradykinesia in early Huntington's disease. Neurology. 2000;54(1):119-25.

[11] Neuroleptic. Malignant Syndrome with Aripiprazole in Huntington's Disease. Gahr M, Orth M, Abler B. Mov Disord. 2010;25(14):2475-6.

[12] Srivastava A, Borkar HA, Chandak S. Olanzapine-induced neuroleptic malignant syndrome in a patient with paranoid schizophrenia. Psychiatry and Clinical Neurosciences. 2009;63:119-21. 